\title{
A spiking-neuron model of memory encoding and replay in hippocampus
}

\author{
Oliver Trujillo*, Chris Eliasmith \\ From The Twenty Third Annual Computational Neuroscience Meeting: CNS*2014 \\ Québec City, Canada. 26-31 July 2014
}

Hippocampal cells replay sequences of neural activity that have been experienced in the past, implicating the hippocampus in episodic memory. We present a spiking-neuron attractor network model that can encode arbitrary experiences in a combination of learned connection weights and neural activity. The model can then recall part or all of the encoded experience based on contextual cues, simulating hippocampal replay.

The simulation is run using the Neural Engineering Framework (NEF) [1] and the Nengo neural modelling tool [2]. It consists of 77740 simulated spiking LIF neurons, divided into populations representing hippocampal areas CA3 and CA1, and part of EC. The NEF provides a method for dynamically representing vectorvalues and computing transformations on them with neural populations.

Our model takes as input a sequence of high-dimensional vectors representing sensory information entering the hippocampus from EC. These vectors can represent position information (place cells) or any other form of processed sensory data. Strong recurrently connected populations in area CA3 cycle through a series of temporal

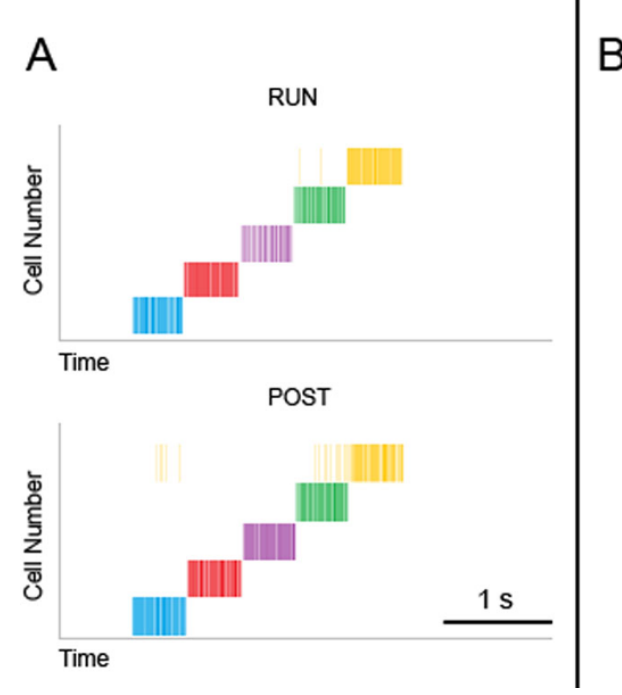

B

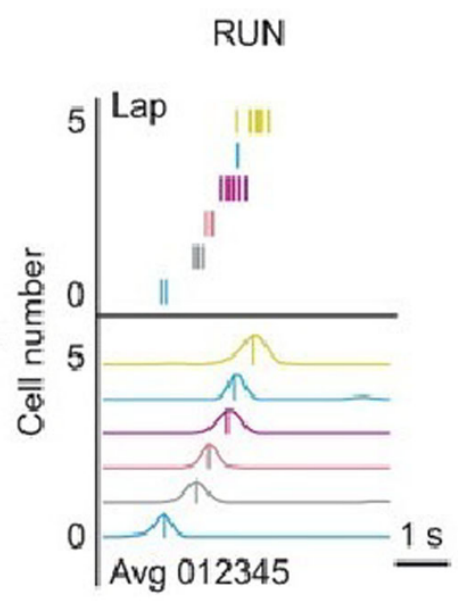

Figure 1 Neural spike data collected from the model (A) and rats (B) showing similar neural spike data during the experiment (RUN) and during recall $(\mathrm{POST})$.

\footnotetext{
* Correspondence: otrujill@uwaterloo.ca

Centre for Theoretical Neuroscience, University of Waterloo, Waterloo,

Ontario, Canada N2L 3G1
} 
indices, and a Hebbian learning rule allows for these indices to be uniquely generated for a given environment. Using the NEF to compute a binding operator [3], the network associatively binds the sensory input vectors with an index. These bindings are stored in the neural activity of the recurrent CA1-EC loop. The network can switch between encoding and recall modes, and when in recall mode can look up the corresponding index bound to the experience being recalled and, using the same indexing system in CA3, can complete the pattern of neural activity corresponding to its previously remembered experience.

The model was run in encoding mode on a sequence of 5 different random HRR vectors in 64 dimensions over a period of 2.5 seconds. It was then given the first in the sequence of vectors as input. After 1 second of re-orientation, the model was switched into recall mode. Figure 1A shows spike trains from neurons in the model at a point in the original run and at the corresponding point in the recalled sequence. For comparison, figure 1B shows hippocampal recordings from rodents in [4].

To the best of our knowledge, this is the first spiking neural model to take into account both timing and sensory tuning of hippocampal cells, while exhibiting the ability to recall previously experienced sequences. In addition, it operates on arbitrary sensory vectors as input, thus not constraining the model to spatial or non-spatial information and allowing it to be extended to perform spatial navigation tasks.

\section{Acknowledgements}

Air Force Office of Scientific Research grant FA8655-13-1-3084, Canada Research Chairs, NSERC Discovery grant, Canadian Foundation for Innovation, Ontario Innovation Trust, NSERC Postgraduate Scholarship.

Published: 21 July 2014

\section{References}

1. Eliasmith C, Anderson CH: Neural Engineering The MIT Press; 2004.

2. Nengo. [http://www.nengo.ca].

3. Plate TA: Holographic reduced representations. Neural networks, IEEE transactions 1995, 6(3):623-641.

4. Ji D, Wilson MA: Coordinated memory replay in the visual cortex and hippocampus during sleep. Nature neuroscience 2007, 10(1):100-107.

doi:10.1186/1471-2202-15-S1-P166

Cite this article as: Trujillo and Eliasmith: A spiking-neuron model of memory encoding and replay in hippocampus. BMC Neuroscience 2014 15(Suppl 1):P166.

\section{Submit your next manuscript to BioMed Central} and take full advantage of:

- Convenient online submission

- Thorough peer review

- No space constraints or color figure charges

- Immediate publication on acceptance

- Inclusion in PubMed, CAS, Scopus and Google Scholar

- Research which is freely available for redistribution

Submit your manuscript at www.biomedcentral.com/submit 